\title{
Expression of the RON receptor tyrosine kinase and its association with gastric carcinoma versus normal gastric tissues Donghui Zhou*1, Gang Pan ${ }^{1}$, Chen Zheng1, Jingjing Zheng ${ }^{2}$, Liping Yian ${ }^{1}$ and Xiaodong Teng 1
}

\author{
Address: ${ }^{1}$ Department of oncology, The First Affiliated Hospital of College of Medicine of Zhejiang University, Hangzhou, 310003 , PR China and \\ ${ }^{2}$ Department of Surgery, Lishui Central Hospital, Lishui, 323000, PR China \\ Email: Donghui Zhou* - zdh19838@hotmail.com; Gang Pan - xjxsh@163.com; Chen Zheng -wentao39@163.com; \\ Jingjing Zheng - drzjj@hotmail.com; Liping Yian - yingyingmama1976@yahoo.com.cn; Xiaodong Teng - tengxd@sina.com \\ * Corresponding author
}

Published: 28 November 2008

BMC Cancer 2008, 8:353 doi:10.1 186/147/-2407-8-353
Received: 18 June 2008

Accepted: 28 November 2008

This article is available from: http://www.biomedcentral.com//47/-2407/8/353

(c) 2008 Dong-hui et al; licensee BioMed Central Ltd.

This is an Open Access article distributed under the terms of the Creative Commons Attribution License (http://creativecommons.org/licenses/by/2.0), which permits unrestricted use, distribution, and reproduction in any medium, provided the original work is properly cited.

\begin{abstract}
Background: Recepteur d'origine nantais (RON) is a receptor tyrosine kinase that is activated by a serum-derived, macrophage stimulating protein (MSP) growth factor and is expressed in many malignant tumors. The aim of the present study was to reveal the protein expression profile of RON and its relationship with clinicopathological characteristics of gastric carcinoma and prognosis.

Methods: Gastric carcinoma tissue from 98 patients, along with 29 specimens of paraneoplastic tissue and 10 specimens of normal gastric mucosa, were examined by immunohistochemistry (IHC). Western blot analysis of 19 samples of gastric carcinoma tissue and corresponding paraneoplastic tissue, 8 specimens of normal gastric mucosa, and 2 specimens of normal lymph node samples also detected expression of a splice variant of RON, RON $\triangle 165$. All samples obtained were accompanied by patient follow-up data that ranged from 3 to 89 months (median time: 22 months).

Results: The rate of positive RON expression differed significantly between gastric carcinoma tissues [56.1\%, (55/98)] and paraneoplastic tissues [25.6\%, (8/29)] $(p=0.007)$. In contrast, RON expression was absent in normal gastric mucosa samples. RON expression positively correlated with the invasive depth of the tumor $(p=0.019)$, perigastric lymph nodes metastasis $(p=0.019)$, and TNM stage $(p=0.00 \mathrm{I})$. However, RON expression was independent of tumor growth pattern according to Bormann criteria ( $\mathrm{P}$ $=0.209)$, histopathological grade $(p=0.196)$, and incidence of distant metastasis $(p=0.400)$. RON expression was not related to a patient's survival rate $(p=0.195)$. RON $\Delta 165$ was strongly expressed in fresh gastric carcinoma tissue, corresponding paraneoplastic tissue, and perigastric lymph nodes with metastatic carcinoma. In contrast, expression of RON $\Delta \mathrm{I} 65$ was not observed in normal gastric mucosa and normal lymph node tissue samples.
\end{abstract}

Conclusion: RON expression is significant in gastric carcinoma tissue and corresponding paraneoplastic tissue, but is not expressed in normal gastric mucosa. Expression of RON $\triangle 165$ was similarly observed in gastric carcinoma tissue and in metastases present in lymph node tissues. We hypothesize that RON and its splice variant play an important role in the occurrence, progression, and metastasis of gastric carcinoma, and therefore may represent a useful marker to evaluate the biological activity of gastric carcinoma. 


\section{Background}

Morbidity from gastric carcinoma is increasing each year, while the age of onset is decreasing. In China, gastric carcinoma is the top malignant tumor in both categories of morbidity and mortality [1]. Recepteur d'origine nantais (RON) is a receptor tyrosine kinase (RTK) that belongs to the MET proto-oncogene family. Recent studies of a RON knockout mouse model shows that complete disruption of the RON gene is embryonic lethal [2], demonstrating that RON is essential in embryonic development. RON is activated by a serum-derived, macrophage stimulating protein (MSP) growth factor, and studies have shown that RON is expressed in many malignant tumors and plays a role in their occurrence and progression.

Zhou et al. [3] found that RON is strongly expressed in colorectal carcinomas, and they identified three splicing variants of RON (RON $\Delta 160$, RON $\Delta 165$, and RON $\Delta 155$ ). The expression of RON and its variants was associated with the progression of colorectal carcinoma. Other studies have shown that the expression of RON and its splice variants can precipitate colon epithelial cell colony formation and increase their viability. When expression of RON and its splice variants were targeted by siRNA in rectal tumor cell strains, proliferation and metatasis were significantly inhibited, concomitant with increased apoptosis. Based on these data, RON and its splice variants are hypothesized to have an important role in the occurrence, progression, and metastasis of rectal cancer [4]. Similarly, studies of RON expression in both breast and bladder cancer tissues has shown increased levels of RON expression and a correlation with histological grade $[5,6]$.

To our knowledge, the role of RON in gastric cancer has only been studied by Collesi et al [7]. In this study the splice variant of RON, RON $\Delta 165$, was detected in the gastric carcinoma cell strain, KATO-III. RON $\Delta 165$ was shown to enhance the invasion of gastric cancer cells, indicating a role for the splice variant of RON in the malignant transformation of gastric cells to a carcinoma. In this study, we examine the expression of RON in the gastric carcinoma tissue of 98 patients using the Envision immunohistochemistry (IHC) method. To examine expression of RON $\Delta 165$, Western blotting of fresh gastric carcinoma tissue was performed. The patients enrolled in our study were monitored between 3 and 89 months post-operation to investigate the association of RON expression, including its splice variant, to the clinicopathological characteristics of gastric carcinoma and prognosis. Due to the complex pathogenesis of gastric carcinoma, therapeutic efficacy has not proven to be very effective. Thus, it is important to investigate the pathogenesis of gastric carcinoma to find new therapeutic targets.

\section{Methods \\ Patient samples}

Paraffin embedded tissue samples from 98 patients who underwent surgery for pathologically confirmed gastric carcinoma in the First Affiliated Hospital of College of Medicine of Zhejiang University between 1998 and 2003 were obtained. Gastric samples were used with the consent of the patients and permission of the Ethics Committees of Hospitals. Seventy men and 28 women, ranging in age from 21 to 76 years (median age 58 years), were included in this study. Patient diagnosis included 78 cases of sinus ventriculi carcinoma, 16 cases of gastric cardia carcinoma, and 4 cases of total gastric carcinoma. Postsurgery pathological reports diagnosed 2 well-differentiated adenocarcinomas, 21 moderately differentiated adenocarcinomas, 69 poorly differentiated adenocarcinomas, 1 signet-ring cell carcinoma, 2 mucinous adenocarcinomas, and 3 severe dysplasia with cancerization. According to UICCTNM (1998), the histological grade of the samples was determined to include 26 stage I cases, 14 stage II cases, 30 stage III cases, and 28 stage IV cases. The followup rate for our patients was $92 \%$, with 8 patients not available to complete the follow-up. The follow-up period was between 3 and 89 months (median time of 22 months) following surgery. Ten samples of normal gastric mucosa were examined as controls. Nineteen samples of fresh gastric carcinoma tissue, paraneoplastic tissue, and positive perigastric lymph node tissue were collected for Western blot analysis. Ten samples of normal gastric mucosa were also included as controls.

\section{Immunohistochemistry}

Paraffin samples were sectioned into $4 \mu \mathrm{m}$ thick slices which were deparaffinized, hydrated and incubated for 20 min in $0.3 \% \mathrm{H}_{2} \mathrm{O}_{2}$ to inhibit endogenous peroxidase activity. For antigen retrieval, samples were incubated with $0.1 \mathrm{M}$ citrate buffer ( $\mathrm{pH}$ 6.0) and heated in a pressure cooker for $8 \mathrm{~min}$. After rinsing in TBS, the slides were incubated with 3\% normal donkey serum (NDS) in TBS for 20 min to prevent non-specific binding of the first antibody. Anti-RON rabbit monoclonal antibody Ron $\beta$ (C-20) (Santa Cruz Biotechnologies, USA) was used at 1:400. The Envision kit (DAKO) was used according to the manufacturer's directions, and bound antibody was detected using DAB. Samples were also co-stained with hematoxylin. Samples were observed under a light microscope and a PBS-only staining sample was used as a background control.

\section{Evaluation criteria for RON detection by immunohistochemistry}

The assessment of the grade of staining was determined using a blinded evaluation procedure by experienced pathologists. High-power fields $(400 \times)$ using standard light microscopy were divided into ten sections which 
were randomly scored. Tumor cells with buffy particles in the intracytoplasm were scored as positive, and the percentage of positive tumor cells and staining intensity for each sample was recorded. Tumor cells that did not stain for RON expression were scored as negative, cells that weakly stained were scored as 1 , moderate staining was scored with a 2, and strongly staining samples were scored as 3. Of the positive tumor cells detected, $<5 \%$ were negative, between 5 and 24\% were scored as 1, 25-49\% were scored with a 2 , and $>50 \%$ were scored with a 3 . The cumulative score of the tumor cells identified in a sample determined a sample's final score. Based on the final score, the tumor tissue was determined to be negative (score of 0-2), or positive (score of 3-6) for RON expression.

\section{Protein immunoblotting}

Fifty micrograms of total protein for each sample was separated by electrophoresis on a $10 \%$ polyacrylamide gel and transferred to PVDF membrane (Millipore). Detection of RON protein was performed using an anti-RON rabbit monoclonal antibody Ron $\beta$ (C-20) (Santa Cruz Biotechnologies, USA) (1:400). Antibody binding was visualized using an anti-rabbit antibody conjugated to horseradish peroxidase and an Enhanced chemiluminescence kit (ECL) (Biological Industries, Inc. Israel). $\beta$-actin was used as a loading control.

\section{Statistical analysis}

SPSS 13.0 statistical software was used to identify the statistical significance of our data using fourfold tables or the $\mathrm{R} \times \mathrm{C}$ table $\chi^{2}$ test. Survival curves were evaluated using the Kaplan-Meier method and comparisons of survival rates were tested using a Log-rank test.

\section{Results}

RON expression in gastric tissue and paraneoplastic tissue RON protein was observed in the cytolymph, but not in the nucleolus, of gastric carcinoma cells and typically appeared as a buffy granulo-staining (Fig. 1). The percentage of positive tumor cells and the staining intensity for each sample was recorded. For the 98 patient samples evaluated, RON expression was observed in $56.1 \%$ of the gastric carcinoma tissues (55/98), but not in samples of normal gastric mucosa. In gastric carcinoma paraneoplastic tissue, which was collected from the gastric mucous layer $0.5 \mathrm{~cm}$ away from the gastric carcinoma specimens, RON protein was observed in $25.6 \%$ of samples $(8 / 29)$. These data represent a significant statistical difference in RON protein expression between gastric carcinoma tissue and its associated paraneoplastic tissue $\left(\chi^{2}=7.290, \mathrm{p}=\right.$ 0.007).

\section{RON protein expression in relation to the pathology of gastric carcinoma}

RON protein expression was not found to be associated with gender, age, or diseased region $(p>0.05)$. However, in gastric carcinoma tissues, the deeper a carcinoma cell infiltrated, the more strongly RON protein was expressed $(p=0.019)$. RON protein expression was also found to positively correlate with perigastric lymph node metastasis and clinical pathology stage with statistical significance $(p=0.019, p=0.001)$ (Table 1$)$. In contrast, there were no significant statistical differences in RON expression with the pathology grading, Borrmann type, or presence of metastasis. However, it was observed that RON expression was stronger in the Borrmann III/IV group (63.6\%) than in the Borrmann I/II group (53.8\%), stronger in the distant metastasis group $(68.2 \%)$ than in the non-distant metastasis group (52.6\%), and stronger in the histologically lower differentiated group (52.4\%) than in the moderately and highly differentiated histology group (39.1\%). There were no significant statistical differences for these comparisons $(\mathrm{p}=0.209 ; \mathrm{p}=0.400 ; \mathrm{p}=0.196)$, respectively (Table 1).

\section{RON expression and survival rates}

For the 90 patients enrolled in this study that were followed post-operationally, no significant statistical difference in survival rates was found between patients with RON-positive gastric carcinoma samples versus RON-negative gastric carcinoma samples (Fig. 2).

\section{Detection of the RON $\triangle 165$ splice variant}

In the 19 samples of fresh gastric carcinoma tissues collected, along with corresponding paraneoplastic tissues, and pathologically confirmed perigastric lymph nodes with metastatic carcinoma, the splice variant of RON, RON $\Delta 165$, was expressed. In contrast, no expression of RON protein was observed in normal gastric mucosa or normal lymph node tissue (Fig. 3, 4).

\section{Discussion}

In the current study, we show by both IHC and Western blot, that RON expression is not detectable in normal gastric mucosa. These observations are consistent with results from Okino et al [8]. However, staining of RON in carcinoma tissues and corresponding paraneoplastic tissues by IHC showed significant expression of RON relative to normal gastric mucosa samples. Western blot analysis provided more specific information regarding RON expression with detection of RON $\Delta 165$ in gastric carcinoma tissues, corresponding paraneoplastic tissues, and lymph nodes with carcinoma metastases. These data indicate that expression of full-length $\mathrm{RON}$, as well as a variant of RON, are expressed during the process of malignant transformation of gastric epithelial cells. Based on these data and work in other studies on the role of RON in tum- 

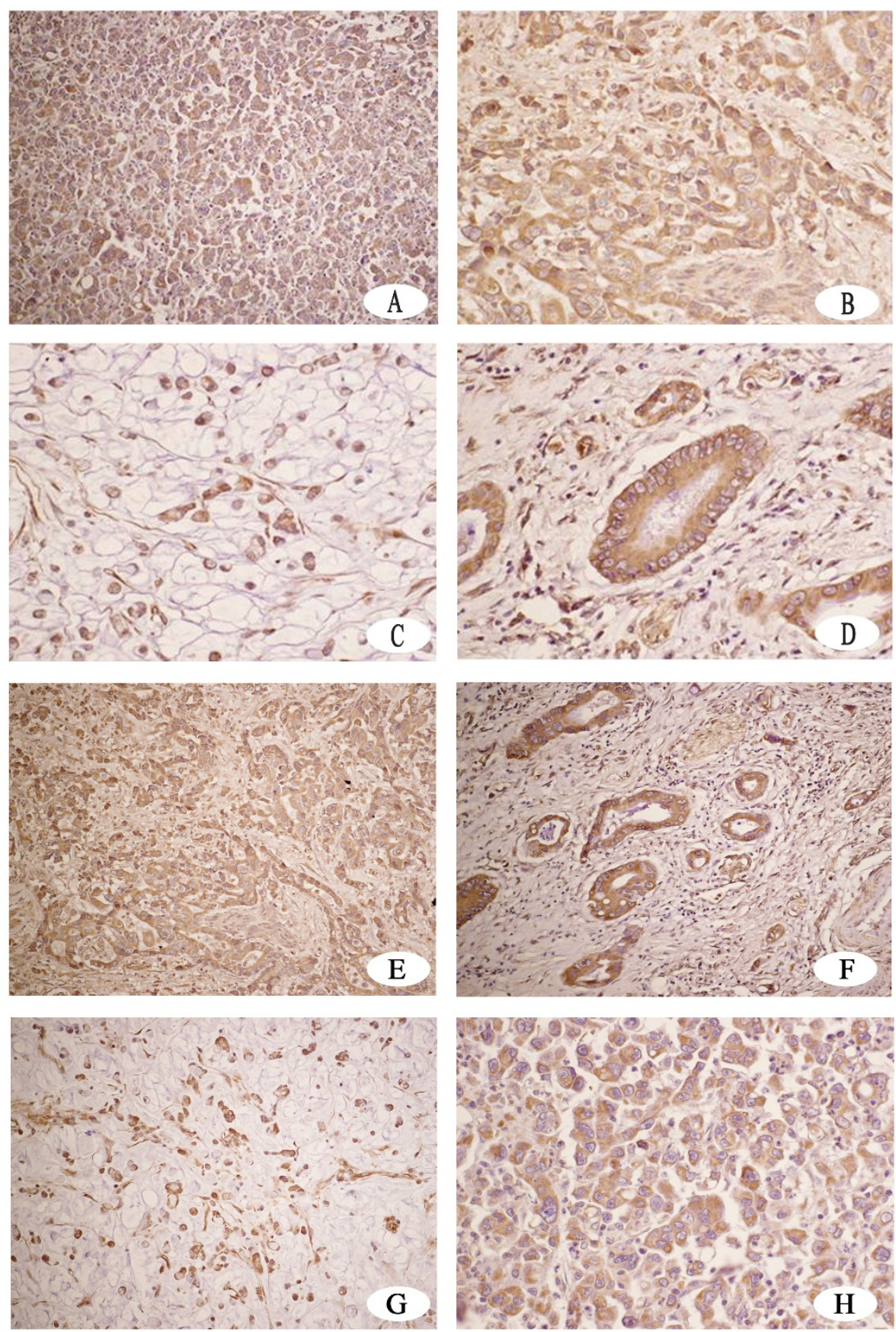

\section{Figure I}

Detection of RON expression by immunhistochemistry. A, H) Detection of RON in a poorly differentiated gastric adenocarcinoma sample. 200x and $400 \times$ magnification. B, E) Magnified view of RON detection in a sample of poorly differentiated gastric adenocarcinoma. $400 \times$ and $200 \times$ magnification. C) Detection of RON in a sample of signet-ring cell carcinoma. 400x magnification. D) Detection of RON in a sample of moderately differentiated gastric adenocarcinoma. 400x magnification. F) Detection of RON in a sample of moderately differentiated gastric adenocarcinoma. 200× magnification. G) Detection of RON in a sample of signet-ring cell carcinoma. $400 \times$ magnification. 
Table I: Relationship of RON expression to pathology parameters

\begin{tabular}{|c|c|c|c|c|}
\hline & $\begin{array}{l}\text { RON } \\
(+)(\%)\end{array}$ & $\begin{array}{c}\text { RON } \\
(-)\end{array}$ & $\mathrm{X}^{2}$ & $P$ \\
\hline \multicolumn{5}{|l|}{ Invasive Depth } \\
\hline Early gastric cancer & $5(29.4)$ & 12 & & \\
\hline Infiltrated to muscular layer & $15(5 \mid .7)$ & 14 & 7.795 & $p=0.019$ \\
\hline Infiltrated to serous membrane & $35(67.3)$ & 17 & & \\
\hline \multicolumn{5}{|l|}{ TNM Stage } \\
\hline Stage & $14(35.0)$ & 26 & 12.245 & $p=0.001$ \\
\hline Stage & $4 \mathrm{I}(70.7)$ & 17 & & \\
\hline \multicolumn{5}{|l|}{ Metastases to Lymph Nodes } \\
\hline $\mathrm{N}_{0}$ & II (37.9) & 18 & & \\
\hline $\mathrm{N}_{\mathrm{I}-3}$ & $44(63.8)$ & 25 & 5.535 & $P=0.019$ \\
\hline \multicolumn{5}{|l|}{ Borrmann Grade } \\
\hline I/II group & I 4 (53.8) & 12 & & \\
\hline III/IV group & $35(63.6)$ & 20 & 0.708 & $p=0.400$ \\
\hline Others & 6 & 11 & & \\
\hline \multicolumn{5}{|l|}{ Histology Grade } \\
\hline Moderately \& highly differentiated & $9(39.1)$ & 14 & 1.577 & $p=0.209$ \\
\hline Less differentiated $*$ & $39(54.2)$ & 33 & & \\
\hline Others** & 0 & 3 & & \\
\hline \multicolumn{5}{|l|}{ Distant Metastasis } \\
\hline$M_{0}$ & $40(52.6)$ & 36 & 1.675 & $P=0.196$ \\
\hline$M_{1}$ & $15(68.2)$ & 7 & & \\
\hline
\end{tabular}

*Includes poorly differentiated adenocarcinoma, signet-ring cell carcinoma, mucinous adenocarcinoma; ** Severe dysplasia with cancerization.

origenic phenotypes, we hypothesize that RON may act as an oncogene to promote the occurrence, progression, invasion, and metastasis of gastric cancer.

Previous studies have shown that expression of RON correlates with the invasion of tumor cells. Maggiora et al [9] reported that RON was expressed in 55\% of ovarian can-

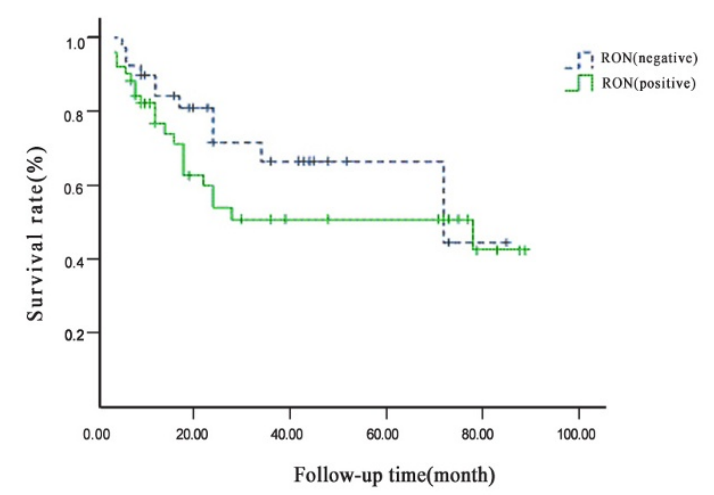

Figure 2

Survival curve for RON+ versus RON-patients. cer tissues. Furthermore, if an ovarian cancer cell line that strongly expresses RON is induced by MSP, tumor growth and invasion is significantly enhanced. Based on these data, it was hypothesized that RON plays a role in the progression of ovarian cancer. Similarly, Chan et al [10] showed that expression of RON promoted the growth and malignant transformation of skin papillomas, and Cheng et al [6] showed that strong expression of RON in bladder cancer cell lines correlated with increased proliferation and apoptosis inhibition. The latter study also demon-

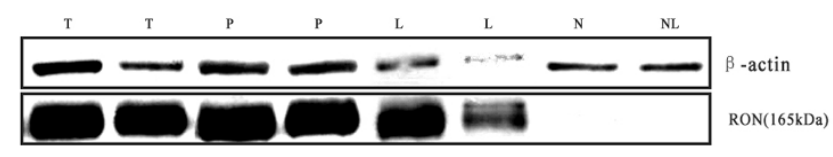

Figure 3

Western blot analysis of RON expression in gastric carcinoma tissue, paraneoplastic tissue, and perigastric lymph nodes. Duplicate samples of gastric carcinoma tissue $(T)$, paraneoplastic tissue $(P)$, and metastatic lymph node $(\mathrm{L})$ tissues were analyzed for expression of RON $\Delta$ I65 (165 kDa). Normal gastric mucosa (N) and normal lymph node (NL) tissue samples were compared. $\beta$-actin (44 kDa) was used as a loading control. 


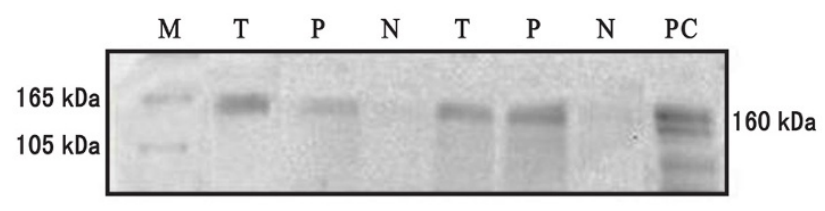

Figure 4

Western blot analysis of RON expression in gastric carcinoma tissue, paraneoplastic tissue, and normal gastric mucosa. Duplicate samples of gastric carcinoma tissue $(T)$ and paraneoplastic tissue $(P)$ were analyzed for expression of RON $\Delta$ I65 (I65 kDa) and compared with normal gastric mucosa $(N)$ tissue samples. Marker $(M)$ and positive control $(\mathrm{PC})(160 \mathrm{kDa})$ were included.

strated that the expression of RON in gastric carcinoma positively correlated with the invasive depth of the gastric cancer cell and lymph nodes metastasis, and the level of expression increased significantly with higher clinical pathologies of gastric cancer. Therefore, strong expression of RON in gastric cancer tissues may serve as a marker to indicate a conversion of normal cells to a malignant phenotype, which associated with increased tumor cell invasion and metastasis, would be associated with a poor prognosis for long-term survival. The proximity of gastric cancer tissue to the epithelial dysplasia of the stomach results in the susceptibility of the epithelial dysplasia of the stomach to precancerous lesions. Therefore, by reporting the expression of RON in paraneoplastic tissue, which was collected $\sim 0.5 \mathrm{~cm}$ away from the gastric carcinoma samples, we are providing data to support our hypothesis that RON plays an important role in promoting gastric cancer and may serve as a marker for the conversion of tissues to a malignant phenotype.

In previous studies looking at the relationship between RON expression and survival rates, Lee et al [11] found that MET and RON were independent prognostic factors of long-term recurrence of breast cancer, and that specimens positive for MET and RON expression had a significantly lower 10-year disease-free survival rate. Similarly, Cheng et al [6] reported a lower survival rate for bladder cancers positive for both MET and RON. In contrast, we found that the survival rate of patients positive for RON expression was not lower than the survival rate for the RON-negative patient group. It is possible that the survival rate of patients with gastric cancer can be affected by many factors, and our follow-up data was not sufficient to provide additional insight into which factors might be important.

\section{Conclusion}

The results of this study show that RON is expressed in gastric cancer, but not in normal gastric mucosa. Further- more, the expression rate of RON in gastric cancer positively correlates with the invasive depth, the clinical pathologic stage, and extent of lymph node metastasis. We hypothesize that RON plays an important role in the occurrence, progression, invasion, and metastasis of gastric cancer, and therefore could be an important marker in assessing the biological behavior of gastric cancer. The results of our studies provide a foundation from which to further identify the mechanistic details of the role of RON in gastric cancer in order to identify RON as a therapeutic target in gastric cancer.

\section{Competing interests}

The authors declare that they have no competing interests.

\section{Authors' contributions}

ZDH carried out study design, final data analysis, drafted the manuscript and revisions to the manuscript. PG, ZC and ZJJ participated in the study concept and the primary data analysis. YLP and TXD performed the IHC analysis. PG was responsible for the Western blot analysis. All authors read and approved the final manuscript.

\section{Acknowledgements}

This work was funded by the Scientific Research Grant of the Health Bureau of Zhejiang Province (No. 491010-WI0380) (No. 2006B039) and Natural Science Foundation of Zhejiang Province, China (No. Y2080 I68).

\section{References}

I. Sun XD, Mu R, Zhou YS, Dai XD, Zhang SW, Huangfu XM, Sun J, Li LD, Lu FZ, Qiao YL: Analysis of mortality rate of stomach cancer and its trend in twenty years in China. Zhonghua Zhong Liu Za Zhi 2004, 26(I):4-9.

2. Muraoka RS, Sun WY, Colbert MC, Waltz SE, Witte DP, Degen JL, Friezner Degen SJ: The RON/STK receptor tyrosine kinase is essential for peri-implantation development in the mouse. J Clin Invest 1999, I 03: I 277-I 285.

3. Zhou YQ, He C, Chen YQ, Wang D, Wang MH: Altered expression of the RON receptor tyrosine kinase in primary human colorectal adenocarcinomas: generation of different splicing RON variants and their oncogenic potential. Oncogene 2003, 22(2):।86-197.

4. Xu XM, Wang D, Shen Q, Chen YQ, Wang MH: RNA-mediated gene silencing of the RON receptor tyrosine kinase alters oncogenic phenotypes of human colorectal carcinoma cells. Oncogene 2004, 23(52):8464-8474.

5. Maggiora P, Marchio S, Stella MC, Giai M, Belfiore A, De Bortoli M, Di Renzo MF, Costantino A, Sismondi P, Comoglio PM: Overexpression of the RON gene in human breast carcinoma. Oncogene 1998, 16:2927-2933.

6. Cheng HL, Liu HS, Lin YJ, Chen HH, Hsu PY, Chang TY, Ho CL, Tzai TS, Chow NH: Co-expression of RON and MET is a prognostic indicator for patients with transitional-cell carcinoma of the bladder. Br J Cancer 2005, 92(1 0): 1906-1914.

7. Collesi C, Santoro MM, Gaudino G, Comoglio PM: A splicing variant of the RON transcript induces constitutive tyrosine kinase activity and an invasive phenotype. Mol Cell Biol 1996, I 6(10):55 I8-5526.

8. Okino T, Egami H, Ohmachi H, Takai E, Tamori Y, Nakagawa A, Nakano S, Sakamoto O, Suda T, Ogawa M: Immunohistochemical analysis of distribution of RON receptor tyrosine kinase in human digestive organs. Dig Dis Sci 200I, 46(2):424-429.

9. Maggiora P, Lorenzato A, Fracchioli S, Costa B, Castagnaro M, Arisio R, Katsaros D, Massobrio M, Comoglio PM, Flavia Di Renzo M: The RON and MET oncogenes are co-expressed in human ovar- 
ian carcinomas and cooperate in activating invasiveness. Exp Cell Res 2003, 288(2):382-389.

10. Chan EL, Peace BE, Collins MH, Toney-Earley K, Waltz SE: Ron tyrosine kinase receptor regulates papilloma growth and malignant conversion in a murine model of skin carcinogenesis. Oncogene 2005, 24(3):479-488.

II. Lee WY, Chen HH, Chow NH, Su WC, Lin PW, Guo HR: Prognostic significance of co-expression of RON and MET receptors in node-negative breast cancer patients. Clin Cancer Res 2005, I I(6):2222-2228.

\section{Pre-publication history}

The pre-publication history for this paper can be accessed here:

http://www.biomedcentral.com/1471-2407/8/353/pre pub

Publish with Bio Med Central and every scientist can read your work free of charge

"BioMed Central will be the most significant development for disseminating the results of biomedical research in our lifetime."

Sir Paul Nurse, Cancer Research UK

Your research papers will be:

- available free of charge to the entire biomedical community

- peer reviewed and published immediately upon acceptance

- cited in PubMed and archived on PubMed Central

- yours - you keep the copyright

Submit your manuscript here:

http://www.biomedcentral.com/info/publishing_adv.asp 